\title{
Regard
}

\section{Gérer la Nature}

\section{Laurent Piermont}

Président directeur général, Société forestière de la Caisse des dépôts, 102 rue Réaumur, 75002 Paris, France

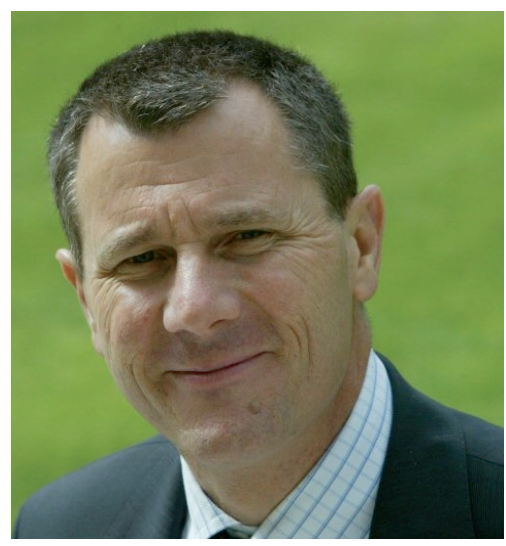

L'idée s'impose avec de plus en plus de force : il est nécessaire de gérer la Nature pour la protéger ${ }^{1}$. La loi forestière française en prend acte puisque, votée en 2001, elle substitue l'objectif de gestion durable à celui de production pour les forêts du pays. En fait, le concept de protection, reposant d'abord sur la constitution de sanctuaires, a montré ses limites : la Nature, "laissée à elle-même», n'évolue pas nécessairement comme nous pourrions l'espérer. Les savanes protégées de Madagascar dont on a éliminé les bovins - les seuls ruminants - sont ainsi mises en péril, avec des feux tardifs spontanés par méthanisation naturelle, suite à un pâturage insuffisant ${ }^{2}$.

L'évolution de nos connaissances sur la dynamique des milieux naturels, le constat que peu d'espaces sur la

Auteur correspondant : laurent.piermont@forestiere-cdc.fr

${ }^{1}$ Ce texte est issu des questions rencontrées par la Société forestière de la Caisse des dépôts pour mettre en place des modes de gestion permettant de préserver au mieux la biodiversité. La Société forestière gère 200000 hectares de forêts et d'espaces naturels pour le compte d'institutionnels, de sociétés et de particuliers.

${ }^{2}$ Jacques Weber, Conservation, développement et coordination : peut-on gérer le social? Colloque panafricain, Gestion des ressources naturelles renouvelables et développement durable, Harare, 24-27 juin 1996. planète échappent à l'influence humaine et l'imprévisibilité de nombreux phénomènes nous obligent à gérer, en nous révélant que la simple protection n'est qu'une modalité de la gestion ${ }^{3}$. Or, le mot gestion suppose un pouvoir sur l'objet de cette gestion et renvoie à trois types de questions - sur les objectifs, sur les méthodes et sur les moyens - qu'il convient de distinguer. L'expérience montre que ce découpage fonctionne assez bien et que, malgré leurs interrelations, confondre ces questions génère des déconvenues, par exemple les mésaventures de l'application en France de la directive européenne Natura 2000. Il m'a donc paru intéressant d'utiliser cette distinction pour évoquer la question de la place qui peut être accordée à la technique et aux outils financiers dans la gestion de la Nature. Le point de vue adopté est celui du gestionnaire ${ }^{4}$ : quelqu'un qui ne fixe pas les objectifs mais doit les mettre en œuvre, en utilisant les moyens (notamment financiers) apportés par d'autres.

\section{Sur les objectifs}

La définition des objectifs, on l'a dit, n'est pas du ressort du gestionnaire, qui se contente d'éclairer la décision. Celle-ci se situe dans le champ politique et résulte d'un jeu entre cinq catégories d'acteurs : propriétaires, collectifs locaux, experts, associations (et autres parties prenantes), pouvoirs publics. Le poids de chacun varie

\footnotetext{
3 Ce constat ne retire évidemment rien à l'intérêt scientifique et culturel de maintenir des espaces dans lesquels n'a lieu aucune intervention humaine.

${ }^{4}$ Cette vision du gestionnaire peut être considérée comme réductrice. Ainsi, Laurent Mermet (Stratégie pour la gestion de l'environnement : la nature comme enjeu de société, Paris, L'Harmattan, 1992) estime qu'il s'agit d'une exception et que la gestion effective résulte de l'action d'un ensemble d'acteurs. En fait, même éclatée, la gestion est confrontée aux questions évoquées ici.
} 
selon les pays, les époques et l'objet de la décision (lutter contre l'effet de serre suppose d'autres moyens que gérer les ressources en coquilles Saint-Jacques d'une baie bretonne). La France privilégie plutôt la recherche d'un équilibre entre les cinq catégories. Dans ce cadre, deux catégories d'objectifs attirent l'attention, car leur mise en œuvre est passablement problématique : la préservation des droits de la Nature et le conflit indigène/exotique.

Les droits de la Nature sont d'abord défendus par les tenants, à des degrés divers, de l'écologie profonde. Plusieurs analyses critiques ont présenté les obstacles ou impasses philosophiques d'une telle approche biocentré $^{5}$. Sans revenir sur ces analyses, mon propos est d'examiner les conséquences en termes de gestion d'une approche conférant à la Nature des droits, des besoins ou des valeurs détachés de considérations humaines. Cette approche progresse, en effet, ainsi qu'en témoigne l'arrêt « Regina » rendu le 11 juillet 1996 par la Cour de justice des Communautés européennes : le gouvernement britannique souhaitait distraire, d'une zone de protection des oiseaux de 4681 hectares en création à l'embouchure de la Tamise, un espace de 22 hectares destiné à des aménagements portuaires considérés comme d'intérêt public majeur. Le recours formé par une association de protection des oiseaux a conduit la Cour européenne à indiquer en substance que seuls les critères ornithologiques, à l'exclusion de tout autre, devaient être pris en compte lors du classement et de la délimitation d'un site. Même si l'arrêt est par ailleurs nuancé et expose que les États sont autorisés, pour des raisons économiques d'intérêt public majeur, à prendre des mesures affectant le site, il ouvre la voie à une reconnaissance de droits supérieurs de la Nature.

Cependant, si devait s'imposer l'idée générale, plutôt sympathique, selon laquelle le maintien des écosystèmes constitue un devoir sacré, un problème technique subsisterait : que maintenir? L'objectif général de "protéger la Nature » masque en effet d'autres questions. Par exemple, les cervidés causent plus de dégâts aux chênes qu'aux hêtres : qu'on le veuille ou non, le développement des populations de chevreuils et de cerfs en France favorise le hêtre au détriment du chêne, sauf action de notre part pour contrecarrer cette évolution. Ceci conduit nécessairement à s'interroger sur le type de forêts que nous souhaitons. Veut-on privilégier le chêne ou le hêtre? La réponse n'est pas inscrite dans la Nature, qui ne souhaite rien. On demande le plus souvent la réponse aux gestionnaires, dont ce n'est pas le rôle, ou aux scientifiques, qui ne sont pas seuls concernés et n'ont pas nécessairement d'arguments rationnels pour choisir. En tout cas, la décision se prend et on remarquera que le débat, lors$\mathrm{qu}^{\prime}$ il a lieu pour des zones soustraites à la production,

\footnotetext{
${ }^{5}$ Notamment Dominique Bourg, 1996. Les Scénarios de l'écologie, Paris, Hachette, et Luc Ferry, 1996. Le Nouvel Ordre écologique, Paris, Grasset.
}

se conclut souvent par la recherche d'un paysage rural idéalisé du début du XXe siècle, avec toutefois beaucoup plus de forêt qu'alors. Le «milieu naturel » devient une construction de notre imaginaire.

Le choix exotique/indigène se range plutôt dans la catégorie des débats inextricables. La loi du 2 février 1995 sur la protection de l'environnement, ainsi que diverses chartes de parcs naturels, documents d'objectifs de sites Natura 2000 ou d'autres espaces naturels, interdisent ou recommandent d'éviter l'introduction d'espèces non indigènes ${ }^{6}$. Il s'agit de préserver l'identité de l'écosystème et d'éviter la banalisation des espaces due au brassage des espèces ${ }^{7}$. Le gestionnaire est alors fondé à se demander : où commence l'exotique? Blé, maïs, pommes de terre venus d'Asie ou d'Amérique ont manifestement le droit de rester, ainsi que les mûriers, les abricotiers, les oliviers, les noyers, les merisiers ou les cyprès (entre autres). Le chêne rouge, le robinier et le chêne des marais, pourtant introduits depuis moins de quatre siècles, ne sont pas trop visés, mais le Douglas, acclimaté ici depuis seulement 1870, sent le soufre. On aimerait, dans ce débat, imaginer des solutions telles que celle choisie (pour de tout autres raisons!) par les créateurs du canal du Midi qui, au XVII siècle, plantèrent le platane commun, un hybride du platane d'Amérique, ramené du Nouveau Monde, et de l'Asiatique, implanté en France depuis très longtemps. En tout cas, l'exclusion des essences non indigènes ne peut constituer à elle seule une directive, surtout au moment où le primat accordé à la notion d'espèce vacille pour laisser le premier rôle au gène $e^{8}$, sans compter les difficultés d'application : le vent, les oiseaux porteurs de graines se soucient peu des règlements.

Ces deux questions font ressortir à l'évidence le besoin d'une hiérarchisation plus poussée des objectifs de gestion des espaces et des espèces. Même sur le seul chapitre de la biodiversité, dont la régression est peu discutable, où les listes d'espèces prioritaires et la notion d'espèce «clef de voûte » orientent l'action, il me parait nécessaire d'approfondir notre capacité à choisir. Au-delà, la hiérarchisation d'objectifs de nature très disparate ne me semble pouvoir aboutir à une application concrète que par des mécanismes contractuels entre les parties en présence, dans un cadre politique... Et au cœur de ces mécanismes contractuels, la question financière apparaît évidemment.

\footnotetext{
${ }^{6}$ Par exemple, les objectifs au 22 avril 2003 du site Natura 2000 FC 4301348, "Complexe forestier et ruisseaux sousvosgiens du territoire de Belfort ».

7 Cette loi, dont tous les décrets d'application ne sont pas encore parus, est toutefois prudente (interdiction des espèces à la fois non indigènes et non cultivées) et prévoit des autorisations pour motifs d'intérêt général.

8 Pierre-Henri Gouyon, 2001. Les Harmonies de la nature à l'épreuve de la biologie, Paris, INRA Éditions.
} 


\section{Sur les méthodes}

La maxime traditionnelle des forestiers « imiter la Nature, hâter son œuvre » illustre l'approche de ceux qui traitent avec la Nature. Elle se décline en quelques règles parmi lesquelles celle du rendement décroissant des actions, la nécessité de raisonner de façon systémique et jamais selon des causalités simples, ou de prévoir les rétroactions... Mais, au-delà, et plus fondamentalement, le gestionnaire est surtout confronté de façon très particulière à l'espace et au temps.

L'espace constitue un bon point de départ dans la gestion de la Nature, à condition toutefois de ne pas oublier les dynamiques des milieux, des populations, des communautés et des écosystèmes; toutes sont causes possibles de transformation de l'objet même de la protection. Comment alors définir l'espace naturel? Le plus naturel des espaces comporte une part d'artificiel, le plus artificiel une part de naturel. Comment regrouper dans un même ensemble, la forêt par exemple, les plantations de pins maritimes, les taillis de chênes du Morvan, presque aussi artificiels, et la forêt amazonienne? Il paraît impossible d'établir une limite rationnelle entre ce qui ressort de l'un et de l'autre ${ }^{9}$. Alors, sans doute faudra-t-il se laisser guider par le langage et considérer comme espace naturel la fraction des espaces que notre imaginaire laisse à la Nature : océans, hautes montagnes, marais, déserts, landes, forêts... Tous espaces qui, dans l'imaginaire occidental, relèvent du domaine de la confusion, des métamorphoses, de la magie, des lieux de la fusion primitive entre les composantes de la Nature, des lieux hors de la cité, par définition antérieurs aux institutions. Difficile, à propos de tels lieux, d'adopter la démarche rationnelle nécessaire au processus de gestion. C'est bien l'une des difficultés.

Quel que soit le degré d'artificialisation de ces espaces, se pose nécessairement la question de leur statut de propriété. D'une part, chacun des trois grands modes d'appropriation paraît correspondre à un domaine de meilleure efficacité : le privé pour la diversité des choix et la rentabilité de la production, la communauté pour la préservation de biens locaux, peu ou pas productifs (captages d'eau, alpages... ), l'État et les autorités supranationales pour conduire les politiques de grande ampleur (créer des parcs naturels, protéger le littoral de l'urbanisation, lutter contre l'effet de serre, lutter contre l'érosion des sols). D'autre part, le statut de propriété joue un rôle décroissant, du moins en Europe occidentale, car la loi étend son domaine d'intervention et encadre de plus en plus fermement le propriétaire quel qu'il soit, qui maîtrise de moins en moins les objectifs. Dans ce contexte, la gestion de la Nature est plus concernée par le «droit

\footnotetext{
${ }^{9}$ Cependant, la notion est employée sans plus de discussion. Ainsi, la loi déjà citée du 2 février 1995 interdit l'introduction d'espèces dans le milieu naturel.
}

d'usage » que par le «droit aux fruits » qui a suscité les débats et conflits que l'on sait au cours de l'histoire (l'avenir d'un étang de Brenne, dans l'Indre, dépend plus aujourd'hui des objectifs de gestion qui lui sont assignés que de la personne qui percevra les produits de la pêche).

Or, les objectifs assignés aux espaces naturels sont nombreux et de plus en plus élaborés par les différentes parties prenantes : archéologues, architectes des bâtiments de France, hydrogéologues du service départemental de l'environnement ou de l'agence de bassin, pêcheurs, varappeurs, promeneurs, producteurs de bois, chasseurs, trufficulteurs, apiculteurs, pompiers, protecteurs de telle ou telle espèce en danger, services chargés de la prévention des risques naturels, de la protection des paysages, des dunes, des réserves naturelles. . . Au-delà de ses objectifs, chacun de ces générateurs de directives a sa propre lecture de l'espace. Sans tenir compte des interlocuteurs qui ne disposent que d'un droit d'influence, on recense tout de même aujourd'hui plus de 70 régimes juridiques ${ }^{10}$, parfaitement légitimes et utiles, susceptibles de s'appliquer en France à un espace naturel. Près de la moitié ont moins de 15 ans, et il ne se passe guère d'année sans qu'un nouveau zonage ne vienne s'ajouter à la liste. Face à cet éclatement et à la multiplication des regards différents portés sur les espaces, l'unicité de gestion est sans doute indispensable, du moins si l'on souhaite rationaliser la poursuite des objectifs. Le gestionnaire unique de chaque espace est chargé de transformer en actes la multiplicité des objectifs et directives et d'expliciter auprès des donneurs d'ordre les conséquences de leur choix. Cela aussi conduit à la hiérarchisation, ainsi qu'à des approches contractuelles adaptées au sujet.

Le temps est le second facteur absolument distinctif de la gestion de la Nature. La société pour laquelle je travaille plante ou fait se régénérer naturellement des milliers d'hectares de forêts chaque année. Cela représente, selon les techniques utilisées, des millions ou des dizaines de millions d'arbres. Planter un arbre en espérant qu'il sera transformé en parquet ou en charpente dans 50 ou 100 ans s'apparente à un acte de foi, et, comme tous les forestiers, nous réfléchissons sérieusement avant d'engager une action qui peut déterminer l'occupation d'une parcelle pour si longtemps et prépare une récolte qui aura lieu dans des conditions politiques, économiques et écologiques aujourd'hui inconnues. Que doivent faire les forestiers quand un collectif de plusieurs milliers de scientifiques prévoit que les températures vont vraisemblablement augmenter de plusieurs degrés au cours du prochain siècle?

Le scénario, actuellement annoncé comme le plus probable, indique des hivers plus doux et pluvieux et des étés plus chauds et secs qu'actuellement, ainsi que

\footnotetext{
10 Jean-Marie Barbier, Forêts de France, nos 393 à 399, mai à décembre 1996.
} 
l'occurrence d'événements extrêmes plus fréquents, tels que tempêtes, violents orages, sécheresses prolongées. Face à de telles annonces, les forestiers ont trois attitudes possibles :

- Premièrement, planter dès à présent des arbres adaptés aux conditions climatiques nouvelles. À ma connaissance, personne ne fait pour l'instant ce choix, très difficile à mettre en œuvre concrètement, car on a du mal à imaginer la transition entre le climat d'hier et celui de demain, et ce qui nous est indiqué des futures conditions n'est pas assez précis pour guider l'action.

- Deuxièmement, ne rien faire, ce qui n'est pas nécessairement l'attitude la moins prudente, notamment pour le mélange futaie-taillis qui constitue le gros de la forêt feuillue française. Cette attitude parie implicitement sur la résistance et l'adaptabilité des forêts et conforte le climat actuel, car les forêts créent en partie, localement, leur propre climat. Son risque est de préparer des dépérissements massifs dans quelques décennies, obligeant le moment venu à une transformation (et à un rajeunissement) radicale de la forêt en quelques années.

- Troisièmement, c'est l'attitude intermédiaire que nous avons adoptée : maintenir pour l'essentiel les choix sylvicoles antérieurs et répondre au cas par cas dans le sens des changements annoncés, par exemple, éviter de planter ou de maintenir des essences en limite de zone d'adaptation écologique, raccourcir la durée des révolutions, privilégier en feuillus les mélanges d'essences...

Ce type de choix, notamment le raccourcissement des révolutions et le maintien de la diversité génétique, constitue une réponse imparfaite, par construction, au principal défi lancé à la gestion : dans certaines limites, les phénomènes naturels sont tout simplement imprévisibles.

Le temps organise la gestion selon des cycles bien distincts dont les convergences, ou divergences, sont cruciales. Ainsi, les grandes plantations résineuses installées en France à partir des années cinquante ont été le fruit d'un important effort collectif, peu critiqué à l'époque (avec tout de même quelques notables exceptions), massivement soutenu par les pouvoirs publics, avec pour objectif la réhabilitation de centaines de milliers d'hectares de forêts ruinées, le rétablissement de la balance commerciale du secteur et le soutien de l'activité en zone rurale. Or, au moment où cet effort commence à aboutir, avec l'arrivée à maturité des premières plantations, l'opinion publique semble avoir majoritairement pris en grippe ce type de sylviculture : les forestiers, gestionnaires des espoirs du passé, se retrouvent en décalage avec l'évolution de l'opinion. En fait, ce cycle est l'un des plus courts de ceux dans lesquels doit s'inscrire l'action. Les cycles naturels sont le plus souvent des cycles lents, rapportés à l'échelle humaine, rythmés par des catastrophes très brèves : les unités sont le million ou la dizaine de milliers d'années pour les changements géologiques (10 millions d'années pour le «recyclage» des fonds océaniques), les millénaires et les siècles pour les cycles climatiques. Le pas du temps pour l'évolution des idées est sans doute cinq à dix fois plus court : décennies et siècles. On franchit encore un facteur dix pour passer au temps politique. .. Celui qui décide des objectifs. Puis cette décision est confrontée à deux cycles lents : la rémanence des actions passées (le taux de mercure dans les lacs suédois continuait $\mathrm{d}$ 'augmenter vingt-cinq ans après l'interdiction de ce métal dans l'industrie papetière), et la lenteur de prise d'effet des nouvelles actions (les reboisements de Napoléon III atteignent maintenant leur pleine maturité).

Les gestionnaires, en charge d'actions qui peuvent s'étaler sur plus d'un siècle, ont inventé le plan de gestion, un outil d'abord utilisé en forêt puis sur tous les types d'espaces naturels depuis les années 1990. Ce document, établi sur une unité de gestion pour une durée de cinq à trente ans, présente un état des lieux, les objectifs assignés à l'espace et la description des actions prévues, le tout illustré par des cartes. Le plan qui lui succède établit un bilan des réalisations, analyse les écarts et prolonge la programmation en tenant compte des modifications du contexte et des objectifs. En même temps sont conservées les archives des actions accomplies (le «sommier»). En cas de bouleversement, le plan de gestion est revu. Cette méthode prospective peut être étendue à l'ensemble des problématiques de gestion de la Nature ${ }^{11}$. Elle permet de traiter avec le temps dans des actions dont la durée excède trop largement le temps des individus.

Bien sûr, les plans de gestion et leurs archives associées n'éviteront pas le dépérissement des chênes si le climat persiste à évoluer. Mais ils nous permettent, par un processus empirique essai/correction, d'accompagner le mouvement, en saisissant les opportunités et en adaptant les techniques afin de garder le cap. Dans l'exemple forestier, il s'agira notamment de repérer et de favoriser les individus résistants aux sécheresses estivales et de conduire les peuplements pour qu'ils soient moins gourmands en eau, de façon à assurer une meilleure transition avec les forêts du futur. Les plans de gestion, et la succession de ces plans, apparaissent ainsi comme des lieux de la synthèse entre des objectifs multiples et comme outils du dialogue avec le temps. Ils constituent l'instrument essentiel de la gestion de la Nature. Le concept, d'abord limité aux secteurs productifs (forêt, puis pêche), s'est étendu à la préservation des ressources naturelles

\footnotetext{
11 Jacques Theys, du Centre de prospective et de veille scientifique, ministère de l'Environnement et Université de Paris 1, expose une telle méthode dans Prospective de l'environnement: la nature est-elle gouvernable?, Espaces et Sociétés, no 74-75, 1994.
} 
lorsque l'approche par la gestion a succédé à la protection $^{12}$.

La Société forestière de la Caisse des dépôts a bâti autour du plan de gestion son dispositif dit « de gestion forestière durable », dont l'un des principaux objectifs est de protéger la biodiversité. Il peut se résumer ainsi :

- Une série de règles, issues de dialogues avec chercheurs et associations : limitation de la taille des coupes rases, gestion particulière des ripisylves, maintien d'arbres vieillissants. . .

- Identification de toute espèce ou espace (protégé ou non) ayant une «valeur écologique » (et là se pose la question de la hiérarchie des critères) et adoption de règles de gestion adaptées.

- Inscription de cet ensemble de prescriptions dans le plan de gestion (20 ans en moyenne) de la forêt.

\section{Sur les moyens}

L'état des milieux naturels dépend de l'ampleur des moyens, techniques et financiers, qui leur sont consacrés. La technique est ainsi de plus en plus utilisée pour la conservation de la Nature. En Seine-et-Marne, la vallée de la Seine a été criblée de carrières d'extraction de matériaux durant des décennies. Ces trous d'eau, objets de nombreuses protestations de défenseurs de la Nature, réaménagés par les entreprises conformément à la loi, après l'arrêt des extractions, sont finalement devenus au fil des ans des espaces de grand intérêt écologique. L'aménagement en cours de la Boucle de Guernes, dans les Yvelines, en est une illustration. Sur 35 hectares, sera en effet constituée une collection de 16 biotopes caractéristiques de la richesse biologique du Val de Seine : modelé de terrain concernant $46000 \mathrm{~m}^{3}$ de substrat calcaire ou acide et $5000 \mathrm{~m}^{3}$ de terre végétale, plantation de pins et feuillus sur 8 hectares, réalisation d'un plan d'eau peu profond de 11 hectares pour la nidification des oiseaux, création d'un front de taille propice à la nidification des hirondelles, constitution de collections de formations végétales sauvages... Tout cela requiert une maîtrise technique, des moyens et des budgets importants (apportés par l'Agence régionale des espaces verts et la Compagnie des sablières de la Seine, l'ONF assurant la maîtrise d'œuvre) et permet de créer de toutes pièces un espace écologique remarquable, sur lequel 150 espèces d'oiseaux, dont 28 remarquables, ont pu être observées. Les exemples de ce genre abondent, les plus significatifs émanant des sociétés d'autoroutes et de carriers, dotées

\footnotetext{
12 Une enquête que nous avons menée en 2003 auprès de gestionnaires d'espaces naturels en Europe, Afrique et Amérique du Nord montre un timide démarrage des plans de gestion dans les années 1980, suivi d'un lent développement dans les années 1990, puis d'une généralisation associée à une meilleure maîtrise du concept au début du XXI ${ }^{\mathrm{e}}$ siècle.
}

de moyens importants et d'obligations réglementaires, recourant à une panoplie de plus en plus large, avec utilisation de textiles biodégradables, de fascines végétales, de plantes sélectionnées. . . Les techniques ainsi mises en œuvre, dites de "génie écologique », se développent : il est tout à fait significatif qu'une recherche Internet lancée en 2004 sur cette appellation aboutisse à plus de 49000 résultats (13000 en 2003), dont beaucoup sont pertinents.

$\mathrm{D}^{\prime}$ un point de vue financier, on retiendra qu'un pont léger, couvert de végétation et permettant aux animaux de traverser une autoroute, coûte aujourd'hui un million d'euros. À plus grande échelle, sur les 60,5 millions d'hectares couverts en 2003 par le réseau Natura 2000, les coûts liés à l'application des directives européennes «Habitats faune flore » et «Oiseaux » atteignent de 3,4 à 5,7 milliards d'euros par an, soit 56 à $94 € /$ ha/an ${ }^{13}$ (ces montants n'incluent pas les coûts du renoncement à la production qu'entraînent, sur certains de ces espaces, les directives de gestion Natura 2000). Autre exemple, le coût pour les 38 pays industrialisés qui ont pris l'engagement de ramener en 2008 leurs émissions de gaz à effet de serre au niveau de ce qu'elles étaient en 1990, varierait, selon les scénarios, de 2 à 7 milliards d'euros ${ }^{14}$.

Évoquer les moyens conduit presque inévitablement à la question de la mise en marché de la Nature. Cette mise en marché, choquante à bien des égards et qui ne date pas d'hier, gagne inexorablement les biens publics les plus inaliénables et semble happée, elle aussi, par le carrousel des instruments financiers. On explique qu'en économie de marché, la production de biens publics est obérée par le comportement des agents économiques qui agissent en «passagers clandestins » en consommant abusivement les ressources publiques pour bénéficier de ce qu'elles ne coûtent rien. La solution serait alors de laisser le marché attribuer un prix à ces ressources afin de les préserver ou de financer leur reconstitution. Cette mise en marché est d'autant plus aisée qu'elle concerne des biens substituables pour lesquels existe une demande solvable.

Mais les biens naturels sont-ils substituables? Une partie de la réponse se trouve dans la nature des biens eux-mêmes. Par exemple, le $\mathrm{CO}_{2}$ est le prototype du bien substituable pour lequel existe une demande solvable. Rien ne distingue, dans l'atmosphère, une molécule de $\mathrm{CO}_{2}$ émise par une centrale électrique américaine, une plâtrerie chinoise ou un feu de forêt indonésien. C'est pourquoi le protocole signé à Kyoto en 1997 a pu imaginer la mise en place d'un marché mondial du $\mathrm{CO}_{2}$, dont l'ampleur pourrait atteindre, en 2008, 60 milliards de

\footnotetext{
13 Lettre d'information Natura 2000, no 17, janvier 2004. Direction générale de l'environnement. Commission européenne, Bruxelles.

14 Mission Climat de la Caisse des dépôts et Arthur Andersen, 2001.
} 
dollars ${ }^{15}$. Le marché du $\mathrm{CO}_{2}$ constitue aussi une réponse efficace $^{16}$ à une demande solvable voulant résoudre un problème exigeant des moyens financiers gigantesques : dès lors que l'on souhaite lutter contre l'effet de serre, il vaut mieux orienter les moyens, limités par définition, vers les actions dont le coût marginal est le plus faible. Or, économiser l'émission d'une tonne de $\mathrm{CO}_{2}$ coûte de 2 à 200 euros selon les secteurs d'activité et les pays. Bien entendu, une solution plus satisfaisante serait de limiter, par voie réglementaire, l'émission de $\mathrm{CO}_{2}$ par individu. Elle serait sans doute moins efficace. C'est pourquoi la Caisse des dépôts a décidé d'engager un programme d'investissement de $25 \mathrm{M} €$ permettant d'acquérir des quotas ou crédits d'émission de $\mathrm{CO}_{2}$ afin de contribuer à la lutte contre l'effet de serre et à la mise en place de ce marché en Europe.

Mais la substituabilité va bien au-delà de l'air et de l'eau. Elle peut concerner de nombreux biens naturels ou, du moins, la représentation que nous en avons : un étang, une forêt, un marais, détruits par une infrastructure, peuvent être reconstitués pour retrouver l'ensemble des caractéristiques et services qu'ils offraient. Cette combinaison de la substituabilité des biens naturels et de l'importance des moyens financiers nécessaires à leur gestion ouvre la voie à la mise en marché de la Nature, et particulièrement à l'utilisation des techniques financières. En effet, comme pour le $\mathrm{CO}_{2}$, dès lors que le cours d'une rivière peut être déplacé sans que ses fonctions écologiques soient altérées, et qu'en même temps les capacités financières sont limitées, on comprend que l'introduction des mécanismes de marché puisse devenir un des enjeux de la période à venir. Parmi les applications les plus immédiates d'une telle évolution, on pourrait ainsi imaginer la création d'un fonds d'intervention pour la biodiversité, alimenté notamment par ceux qui modifient les milieux naturels, chargé de financer la gestion de la biodiversité.

La Société forestière de la Caisse des dépôts a engagé, en 2004, une étude-action sur la création d'un tel fonds en France, en accord avec le ministère de l'Écologie, l'Institut français de la biodiversité et le Centre national du développement durable. Ce fonds pourrait s'inspirer, notamment, de l'expérience nord-américaine : la loi américaine demande en effet que soient compensés ou réparés les dommages causés à l'environnement, mais permet que l'obligation de compenser soit transférée. Cela a contribué au développement d'une activité de financement des mesures compensatoires, qui inclut des systèmes de crédit apparemment efficaces, mais surtout sur des quantités mesurables (destruction d'un hectare de marais, compensée par la création de un ou plusieurs hectares de marais), et se heurte aux difficultés d'évaluation des fonctions écologiques ${ }^{17}$.

\section{Pour conclure : Artémis dévoilée}

Artémis, la déesse grecque de la Nature et de la fertilité, possédait de nombreux espaces naturels sacrés correspondant notamment aux forêts vierges qui s'étendaient au-delà des limites de la cité et des champs cultivés. Invisible, intangible, énigmatique, cruelle, elle régnait sur les contrées sauvages inaccessibles à l'homme. Or, dans ses Métamorphoses, Ovide raconte ainsi le mythe grec d'Actéon : à l'issue d'une chasse, celui-ci s'écarte de sa meute et de ses amis pour se promener au pied d'une montagne. En cet endroit, dans un bois de cyprès et de pins consacrés à Artémis, une fontaine s'écoule dans un bassin limpide. Artémis s'y baigne, en compagnie de ses nymphes. Déjà dévêtue, elle a tendu ses armes à ses suivantes et pénétré dans le bassin quand Actéon la surprend. Artémis, que ses compagnes tentent en vain de protéger du regard d'Actéon, plonge la main dans le bassin et jette de l'eau au visage du chasseur en lui disant : «maintenant, vas raconter que tu m'as vue sans voile; $s i$ tu le peux, j'y consens ». Ce qu'Actéon ne pourra faire, car il se transforme en cerf, perd l'usage de la parole et, poursuivi par ses chiens et ses amis dont il ne peut se faire reconnaître, meurt férocement déchiqueté ${ }^{18}$.

Nous avons dévoilé Artémis, puis nous sommes mis en tête de la rhabiller et même de décider où et quand elle pourrait reprendre son costume de chasseresse. Ambition énorme, qui suppose plus que des débats sur la couleur des vêtements. La gestion de la Nature exige d'abord des méthodes, une organisation, des moyens structurés et réactifs pour espérer atteindre... quelques objectifs.

\footnotetext{
15 Ken Newcomb, Banque mondiale, 2000.

16 Le marché du $\mathrm{SO}_{2}$, lancé aux États-Unis dans les années 1990, a parfaitement fonctionné, au point que les rejets sont désormais négligeables.
}

\footnotetext{
17 Joshua Bishop. Producing and trading habitat or land development as a source of funding for biodiversity conservation. A review of mitigation and conservation banking in the USA and its implication for global biodiversity conservation, IUCN, 10 may 2003.

18 Robert Harisson, 1992. Forêts. Essai sur l'imaginaire occidental, Paris, Flammarion.
} 